\title{
Rhizobial Adaptation to Hosts, a New Facet in the Legume Root-Nodule Symbiosis
}

\author{
Marion Koch, ${ }^{1}$ Nathanaël Delmotte, ${ }^{1}$ Hubert Rehrauer, ${ }^{2}$ Julia A. Vorholt, ${ }^{1}$ Gabriella Pessi, ${ }^{1}$ and \\ Hauke Hennecke ${ }^{1}$ \\ ${ }^{1}$ Institute of Microbiology, ETH, Zurich, Switzerland; ${ }^{2}$ Functional Genomics Center Zurich, University of Zurich and ETH, \\ Zurich, Switzerland
}

Submitted 28 November 2009. Accepted 6 February 2010.

\begin{abstract}
Rhizobia are able to infect legume roots, elicit root nodules, and live therein as endosymbiotic, nitrogen-fixing bacteroids. Host recognition and specificity are the results of early programming events in bacteria and plants, in which important signal molecules play key roles. Here, we introduce a new aspect of this symbiosis: the adaptive response to hosts. This refers to late events in bacteroids in which specific genes are transcribed and translated that help the endosymbionts to meet the disparate environmental requirements imposed by the hosts in which they live. The host-adaptation concept was elaborated with Bradyrhizobium japonicum and three different legumes (soybean, cowpea, and siratro). Transcriptomes and proteomes in root-nodule bacteroids were analyzed and compared, and genes and proteins were identified which are specifically induced in only one of the three hosts. We focused on those determinants that were congruent in the two data sets of host-specific transcripts and proteins: seven for soybean, five for siratro, and two for cowpea. One gene cluster for a predicted ABC-type transporter, differentially expressed in siratro, was deleted in $B$. japonicum. The respective mutant had a symbiotic defect on siratro rather than on soybean or cowpea. This result demonstrates the value of the applied approach and corroborates the host-specific adaptation concept.
\end{abstract}

Certain $\alpha$ - and $\beta$-proteobacteria, collectively called rhizobia, are soil bacteria which are able to establish a symbiotic interaction with legume plants. This leads to the formation of a specific plant organ, the root nodule. Inside nodules, the bacteria live as nitrogen-fixing endosymbionts (bacteroids) in a protected environmental niche. Rhizobia-legume symbioses develop as a result of a complex molecular interplay between both partners (Broughton et al. 2000; Spaink 2000). The host range of rhizobial species is a trait that allows them to nodulate either a narrow or a broad range of legumes. A rather narrow host range has been described for Sinorhizobium meliloti which induces nitrogen-fixing nodules on only few species of the genera

Corresponding author: Hauke Hennecke; Telephone: +41 44 6323318; Fax: +41 44 6321382; E-mail: hennecke@micro.biol.ethz.ch

G. Pessi and H. Hennecke contributed equally as senior authors.

Data deposition: MS/MS data have been deposited in the PRIDE database (10114-10116, 10099-10104). The microarray data have been deposited in NCBI's Gene Expression Omnibus (GEO) database and are accessible through GEO Series accession number GSE18884.

* The $e$-Xtra logo stands for "electronic extra" and indicates that five supplementary tables are available online.
Medicago, Melilotus, and Trigonella. In contrast, numerous Bradyrhizobium spp. have a broader host range because they nodulate legumes from different families such as Papilionoideae, Mimosoideae, and Phaseoleae (Denarié et al. 1992).

At the beginning of symbiotic events, plant-released phenolic root exudates, also known as flavonoids (Peters et al. 1986), lead to the expression of many of the rhizobial genes (nod) required for nodulation. Induction of nod genes is controlled by the NodD protein, a member of the LysR family of transcription regulators (Schell 1993). Point mutations in nodD may expand the number of recognized flavonoid inducers, thereby extending host range (McIver et al. 1989). Apart from NodD, species-specific transcription factors may be involved in controlling host range. In Bradyrhizobium japonicum, for example, an isoflavonoid-stimulated two-component regulatory system NodVW is important for nodulation of mung bean, cowpea, and siratro but only marginally contributes to eliciting symbiosis with soybean (Göttfert et al. 1990; Loh et al. 1997).

The products of nod genes are enzymes for the biosynthesis of bacterial nodulation (Nod) factors (Denarié et al. 1996). These are lipo-chito-oligosaccharides that elicit a series of physiological and developmental responses on host plants leading to nodule organogenesis (Perret et al. 2000; Madsen et al. 2003). The quality and quantity of chemical modifications at the Nod factor are further important determinants that affect host range (Ehrhardt et al. 1995; Denarié et al. 1996).

Once the establishment of a symbiosis between both partners approaches completion, rhizobia are expected to adapt their physiology to that of the host (Oke and Long 1999). In contrast to the remarkable advances in the area of host specificity, current knowledge about bacterial adaptation to hosts is quite limited, and possible processes associated with adaptation have not been investigated systematically. Certain features in the metabolic diversity of rhizobia are probably crucial for adaptation and survival in nodules of disparate host plants. Only sporadic literature exists in support of this notion. For example, a Rhizobium sp. NGR234 pckA mutant defective in phosphoenolpyruvate carboxykinase was reported to display a host-dependent phenotype (Osteras et al. 1991). More recently, host-dependent expression of Rhizobium leguminosarum bv. viciae hydrogenase activity was proposed to be due to a hostderived substance (Brito et al. 2008), and a host-specific regulation of nif and fix gene expression was shown for R. leguminosarum bv. trifolii (Miller et al. 2007).

The aim of the present study was to systematically find out whether or not the exposure of bacteroids to different host nodule environments is reflected by differential gene expression patterns. Specifically, we analyzed the possible adaptation of B. japonicum during symbiosis with three host plants (soy- 
bean, cowpea, and siratro). We have chosen legumes from two different subtribes of the tribe Phaseoleae to cover the maximal diversity of known host plants for $B$. japonicum. Soybean belongs to the subtribe Glycininae, whereas the other two species, cowpea and siratro, are members of the subtribe Phaseolinae (Lee and Hymowitz 2001; Delgado-Salinas et al. 2006). The transcription profiles were assessed with a previously established microarray technology, using a custom-made Affymetrix GeneChip (Hauser et al. 2007). The results were compared and combined with a complementary gel-liquid chromatography tandem mass spectrometry (LC-MS/MS) proteomics analysis of $B$. japonicum bacteroids. Our study culminated in the discovery of sets of $B$. japonicum adaptation genes that are differentially expressed in each of the three hosts. As a proof of principle, we could show that deletion of a gene cluster that is specifically expressed in siratro nodules produced a significant symbiotic defect only on siratro.

\section{RESULTS AND DISCUSSION}

\section{Transcriptome analysis of $B$. japonicum}

in root nodules of cowpea, siratro, and soybean.

Little is known about the genetic determinants of host-specific bacteroid performance in a persistent root nodule. With

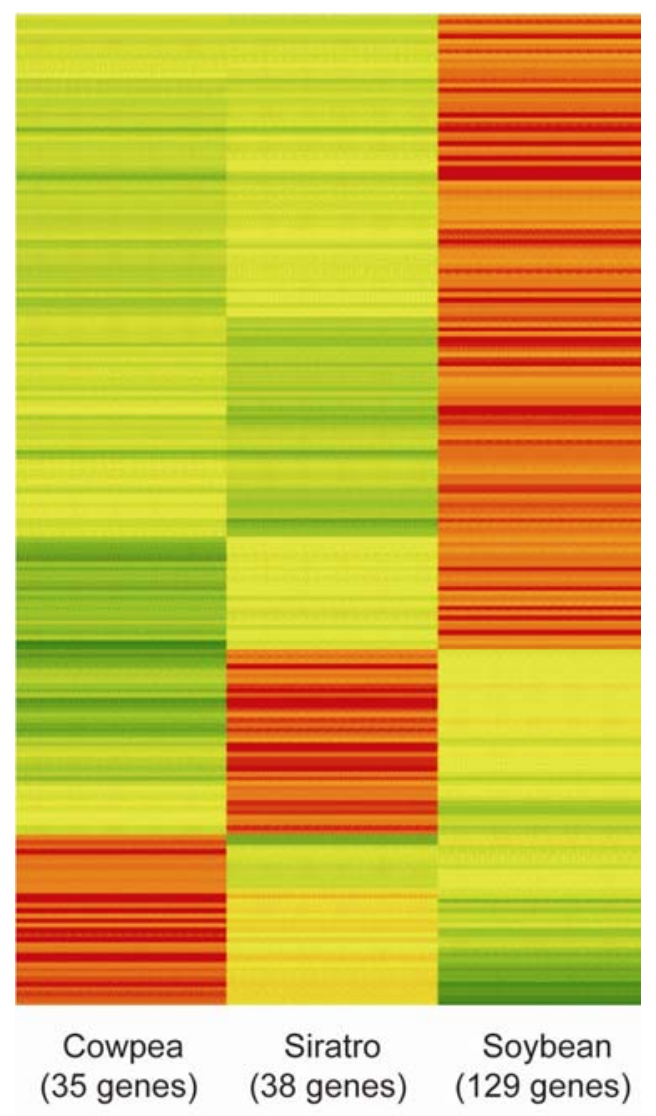

\section{Expression level (log scale):}

$\begin{array}{lllll}0 & 0.5 & 1 & 2 & 5\end{array}$

Fig. 1. Hierarchical clustering of all of the 202 identified host-specific adaptation genes identified by transcriptomics. Gene expression level is shown for Bradyrhizobium japonicum bacteroids at 21 days postinfection (dpi) for soybean and cowpea and $31 \mathrm{dpi}$ for siratro. Color code for the expression level is shown at the bottom; red represents a very high expression and green a very low level of expression (log scale). the aim to monitor possible transcriptional changes in $B$. japonicum cells living in symbiosis with Glycine max (soybean), Vigna unguiculata (cowpea), and Macroptilium atropurpureum (siratro), microarray experiments were performed using a high-density oligonucleotide GeneChip (Hauser et al. 2007; Pessi et al. 2007). RNA from nodules, isolated at the time of maximal nitrogenase activity (discussed below), was processed and analyzed as described (Pessi et al. 2007). At least three independent experiments for each host were carried out and subjected to careful data analysis (discussed below). Although more than 2,000 genes were commonly expressed, regardless of the host, 129 B. japonicum genes were specifically upregulated during symbiotic life in soybean, 38 in siratro, and 35 in cowpea (Supplementary Tables S1, S2, and S3, respectively), which amounts to 202 B. japonicum genes that were defined here as host-specific adaptation genes. A hierarchical clustering analysis of these 202 genes is displayed in Figure 1. The illustration shows a remarkable exclusiveness of their induction.

\section{Proteome analysis of bacteroids \\ from cowpea, siratro, and soybean nodules.}

To identify $B$. japonicum proteins that are detectable in rootnodule symbiosis with soybean, siratro, and cowpea, a sensitive gel LC-MS/MS proteomics approach was applied (Delmotte et al. 2009, 2010). To this end, root nodules were collected at the peak of maximal nitrogenase activity and bacteroids were isolated by gradient centrifugation before they were processed for proteomics (described below). In total, approximately 2,000 proteins were identified in each symbiosis. The method detects most of the expressed soluble proteins and many of the membrane-anchored or -associated proteins but rarely the truly membrane-integral proteins. Among those, 1,620 proteins were detected in bacteroids from all three hosts (Fig. 2), which corresponds roughly to $20 \%$ of the potential B. japonicum proteome as deduced from the genome sequence (Kaneko et al. 2002). Among them, we found almost all of the soluble housekeeping proteins such as DNA and RNA polymerases, aminoacyl-tRNA synthetases, and ribosomal proteins, which can be regarded as an excellent quality control of the method employed. If detection in at least one of three biological replicates is considered, the sensitive proteomics approach identified 238

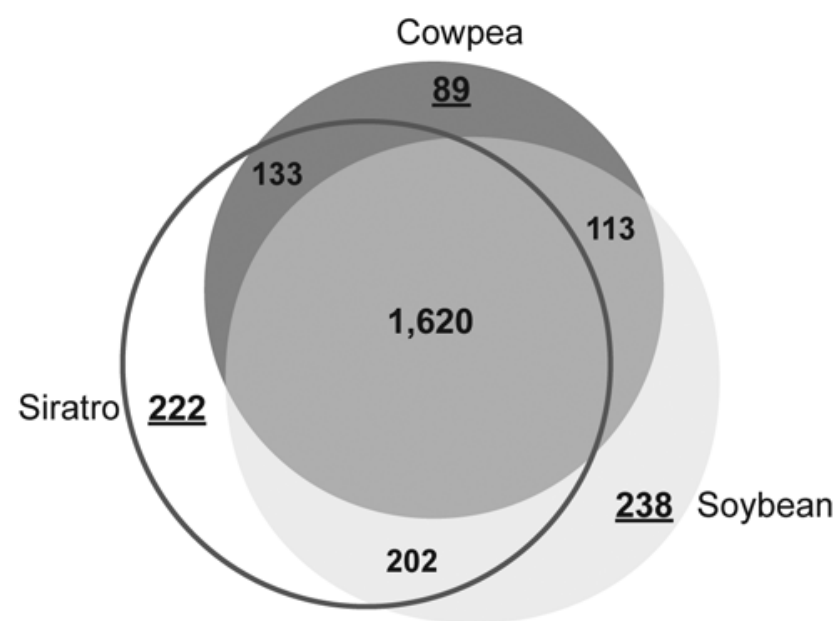

Fig. 2. Venn diagram representing the total number of proteins detected by whole-cell proteomics. Bradyrhizobium japonicum wild-type bacteroids were isolated from cowpea, siratro, and soybean. Protein processing and identification are described in Materials and Methods. In total, 1,620 proteins were identified in all hosts. Host-specific proteins are indicated by underlining. The number of bacteroid proteins shared between just two of the three plants are given in the overlapping sectors of pairwise comparisons. Only unambiguous proteins were considered. 
bacteroid proteins that were detectable only in soybean, 222 in siratro, and 89 in cowpea (Fig. 2). If detection in at least two of three biological replicates is considered, the respective number of proteins drops down to 63 for soybean, 78 for siratro, and 12 for cowpea bacteroids. We assume that, in addition to the stochastic undersampling typical of shotgun proteomics in highly complex protein samples, the primary reasons why proteins are sometimes not detected in all three biological replicates are i) when they are of small size (i.e., the number of peptides available for detection is inherently low), ii) when they are present in low abundance (i.e., they are at a threshold level of detection sensitivity), or iii) a combination of both rea-

A

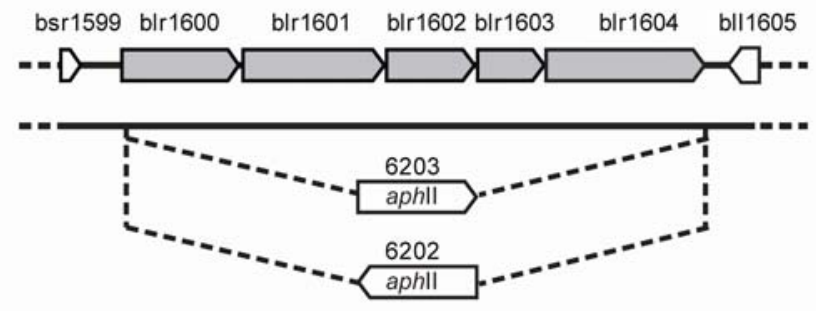

B

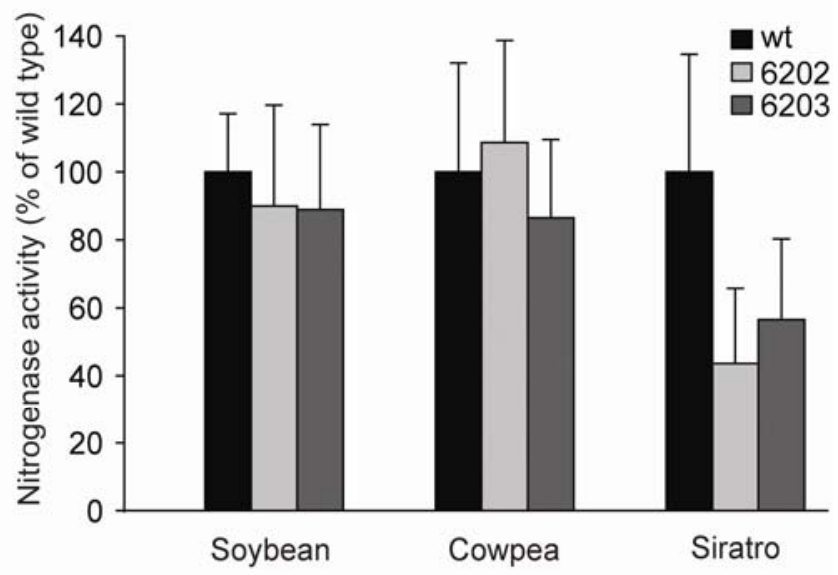

C
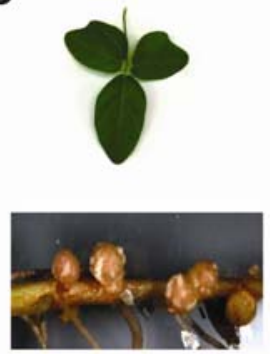

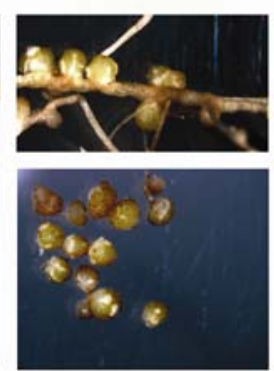

6202
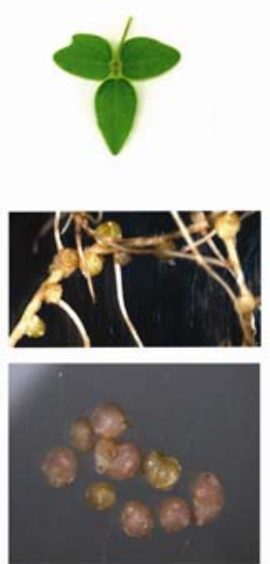

6203
Fig. 3. Genotype and phenotype of $\Delta$ blr1600-1604 mutants. A, Map of the Bradyrhizobium japonicum genomic region expressed specifically in siratro nodules. Gray arrows indicate the blr1600-1604 operon. The orientation of the inserted cassette is given. B, Whole-root-nodule nitrogenase activity assayed by acetylene reduction. Host plants and $B$. japonicum strains are indicated; wt, wild-type. C, Visual inspection of the symbiotic phenotype of siratro plants infected by B. japonicum wt and mutants. sons. Based on the low false discovery rate of our datasets at the spectra level (below 0.02\%) and protein level (below $0.1 \%$ ), almost all protein identifications are assumed to be correct. However, to minimize the risk of overinterpretting our data, a cut-off at a minimum of two hits in the three replicates appears compelling. These concerns will be considered in the so-called stringent data set (discussed below).

Another aspect shown in Figure 2 is how many bacteroid proteins are shared between just two of the three plants. The numbers, however, do not reflect the relatedness of the three hosts (Lee and Hymowitz 2001; Delgado-Salinas et al. 2006).

The stringent data set: where host-specific transcriptomes and proteomes overlap.

We looked for congruence between the list of host-specific adaptation genes identified by transcriptomics and the list of host-specific adaptation proteins found by proteomics. For the latter, proteins were considered only if they were detected in at least two out of three biological replicates. In total, 14 genes were found for which both transcripts as well as proteins are synthesized in a host-specific manner. These are compiled in Table 1, showing two host-specific determinants for cowpea, five for siratro, and seven for soybean. We regard the data set in Table 1 as the most stringent and reliable one in our search for functions involved in the adaptive response. The genes in Table 1 can now be tested for functional involvement in the respective hosts.

\section{An approved determinant}

for bacteroid adaptation to life in siratro nodules.

We focused our attention on the genes that are host-specifically expressed in bacteroids from siratro nodules, and noticed two (blr1601 and bll7011) that encode periplasmic substratebinding proteins associated with ABC-type transporters (Higgins 2001; Moussatova et al. 2008). Being soluble proteins, the blr1601 and bll7011 products were detected by proteomics, whereas the membrane-bound components of the cognate $\mathrm{ABC}$ transporters were not detected. The genes for the latter, however, were found to be siratro-specifically induced just like the genes for the binding proteins, suggesting co-regulation of the contiguous genes and their organization in two separate operons (blr1601-blr1604 and bll7011-bll7008). One of the two operons is depicted in Figure 3A which, in fact, consists of five genes, including blr1600 at the $5^{\prime}$ end. Gene blr1600, which codes for a putative monooxygenase, was also identified as a siratro-specific adaptation determinant by both transcript and protein analysis (Table 1). The tiling-like architecture of the GeneChip (Hauser et al. 2007) allowed us to analyze the transcription profile of the entire blr1600-1604 genomic region, which confirmed that the five genes of the operon are co-transcribed as a polycistronic mRNA. Downstream of the substrate-binding protein gene (blr1601) are two permease genes (blr1602 and blr1603) and the gene for an ATP-binding protein (blr1604).

A rhizobial ABC transporter induced in bacteroids and functioning in a host-specific manner (i.e., in siratro) is a novelty. Therefore, it was necessary to obtain support for such a hostrelated role by mutational analysis. Two $B$. japonicum deletion mutants were constructed by marker replacements (strains 6202 and 6203) in which all five genes of the blr1600-1604 operon were deleted (Fig. 3A). Soybean, cowpea, and siratro plants were then inoculated with the B. japonicum wild-type and the two mutants. Phenotypic analyses revealed that siratro root nodules suffered from a statistically secured $50 \%$ decrease in symbiotic nitrogen fixation activity, as measured in the acetylene reduction test, whereas mutant-infected soybean and cowpea nodules had wild-type activities (Fig. 3B). Moreover, 
only siratro plants inoculated with the mutants showed typical symptoms of limitations such as chlorotic leaves and pale nodules (Fig. 3C). Nodule number and dry weight did not alter when siratro, soybean, and cowpea were infected with the wild-type or the deletion mutant. In conclusion, the blr16001604 operon is specifically important for an optimal function of $B$. japonicum bacteroids in siratro nodules, whereas its presence or absence makes no difference to the symbiotic performance in nodules of soybean and cowpea.

Tentative conclusions regarding the possible function of the blr1600-1604 genes are put forward here, which are solely based on the predicted protein properties and the aforementioned phenotype. The presence of a co-induced periplasmic substrate-binding protein, encoded by blr1601, suggests that the cognate $\mathrm{ABC}$ transporter is an importer rather than an exporter. Hence, siratro nodules are expected to produce a substance that is taken up by $B$. japonicum bacteroids. This could be either a beneficial or a detrimental compound which is either utilized as nutrient or detoxified, respectively. Interestingly, the associated monooxygenase encoded by blr1600 might fulfill either role (i.e., to convert a useful substance into a metabolizable form or to inactivate a toxic compound by oxygenation). The lack of a signal peptide suggests that the monooxygenase is located in the cytoplasm. Intriguingly, it was reported recently that cytoplasmic monooxygenases can be associated with $\mathrm{ABC}$ type transport systems and directly process the substrate after import (Zhang et al. 2007). Although the Blr1601 protein shares $35 \%$ identity with the oligopeptide-binding protein OppA from an $\mathrm{ABC}$ transport system of Xanthomonas axonopodis (Moutran et al. 2007) and carries a solute-binding domain (PF00496) that, in other bacteria, was thought to bind oligopeptides (Tam and Saier 1993), these similarities are not compelling enough to suggest oligopeptides as substrates. Hence, the nature of the transported substrate remains enigmatic.

\section{Further candidates with a perspective to act in a host-responsive manner.}

We restrict ourselves to briefly commenting on just two other promising cases, one again concerning siratro and the other concerning cowpea. i) The siratro-specifically induced operon bll7011-7008 (described above) codes for yet another ABC transporter. Its periplasmic substrate-binding protein (B117011) has been annotated as a sulfonate-binding protein (Kaneko et al. 2002). Therefore, the transporter might be important for the uptake of inorganic or organic sulfur by B. japonicum bacteroids when living in siratro nodules. The need for adequate sulfur supply to bacteroids has recently been addressed in the Lotus japonicus-Mesorhizobium loti symbiosis (Krusell et al. 2005). A host-plant sulfate transporter, SST1, was shown to be important for symbiotic nitrogen fixation and plant development. SST1 was proposed to be inserted into the peribacteroid membrane in which it transports sulfate from the plant cell cytoplasm into the peribacteroid space, thus making sulfate available as a nutrient to bacteroids. ii) The cowpea-specific adaptation genes bl10339, bll0343, and bll0346 (Table 1) map in close vicinity on the B. japonicum genome, and two of the three have been annotated as coding for key enzymes of tyrosine degradation (Kaneko et al. 2002). Our proteomics study has revealed that the product of bl10339, a putative 4-hydroxyphenylpyruvate dioxygenase, is the most abundant of all proteins listed in Table 1, as judged from the number of assigned spectra after LC-MS/MS analysis. By analogy with other work on amino acid utilization (Prell and Poole 2006), it is attractive to speculate that tyrosine is a nutrient for $B$. japonicum bacteroids in cowpea nodules. In this context, it is relevant to note that $S$. meliloti was shown to utilize tyrosine as a carbon and nitrogen source (Milcamps and de Bruijn 1999).

\section{The relaxed data set: transcriptomes and proteomes combined.}

The results of our transcriptomics and proteomics approaches were combined, and all genes and proteins which had been detected as being host-specifically expressed by at least one of the two methods were kept as an expanded, integrated dataset. Thus, we identified 347 genes and proteins for soybean, 253 for siratro, and 120 for cowpea, amounting to a total of 720 potential adaptive-response determinants. There are reasons why entries in the list of transcripts do not appear in the list of proteins or vice versa. For example, an obvious reason has already been alluded to in the case of the $\mathrm{ABC}$ transporters: membrane-integral proteins were missed in the proteome approach, whereas their genes were detected as being host-

Table 1. Bradyrhizobium japonicum host-specific adaptation gene and protein candidates for symbiosis with cowpea, siratro, and soybean assessed by transcriptomics and proteomics

\begin{tabular}{|c|c|c|c|c|c|c|c|}
\hline \multirow{3}{*}{$\frac{\text { Plant, gene }^{\text {b }}}{\text { Cowpea }}$} & \multirow[b]{2}{*}{ Description $^{c}$} & \multirow[b]{2}{*}{ Name $^{d}$} & & & \multicolumn{3}{|c|}{ No. of assigned MS/MS spectra ${ }^{a}$} \\
\hline & & & \multicolumn{2}{|c|}{ Fold change in transcriptomics } & Cowpea & Siratro & Soybean \\
\hline & & & Cowpea vs. soybean & Cowpea vs. siratro & & & \\
\hline bllo339 & 4-Hydroxyphenylpyruvate dioxygenase & $\ldots$ & 3.4 & 8.3 & 126 & 0 & 0 \\
\hline bl10346 & Hypothetical protein & $\ldots$ & 3.2 & 3.4 & 11 & 0 & 0 \\
\hline Siratro & & & Siratro vs. cowpea & Siratro vs. soybean & & & \\
\hline bsr0859 & Hypothetical protein & $\ldots$ & 5.7 & 4.3 & 0 & 4 & 0 \\
\hline blr1600 & Putative monooxygenase & $\ldots$ & 13.6 & 5.1 & 0 & 7 & 0 \\
\hline blr1601 & $\mathrm{ABC}$ transporter substrate-binding protein & $\ldots$ & 7.0 & 5.7 & 0 & 28 & 0 \\
\hline bll6452 & Acyl-CoA dehydrogenase & acd & 9.6 & 7.5 & 0 & 20 & 0 \\
\hline bll7011 & $\mathrm{ABC}$ transporter sulfonate-binding protein & $\ldots$ & 11.5 & 4.1 & 0 & 22 & 0 \\
\hline Soybean & & & Soybean vs. siratro & Soybean vs. cowpea & & & \\
\hline blr1080 & Enoyl-CoA hydratase & $\ldots$ & 9.1 & 7.0 & 0 & 0 & 18 \\
\hline blr1349 & Hypothetical protein & $\ldots$ & 2.7 & 2.9 & 0 & 0 & 7 \\
\hline blr2168 & Putative transketolase $\alpha$ subunit protein & $\ldots$ & 16.1 & 23.1 & 0 & 0 & 28 \\
\hline blr2169 & Putative transketolase $\beta$ subunit protein & $\ldots$ & 11.4 & 8.4 & 0 & 0 & 38 \\
\hline blr2606 & 2-Nitropropane dioxygenase & $\ldots$ & 2.3 & 3.1 & 0 & 0 & 5 \\
\hline blr3389 & $\mathrm{ABC}$ transporter substrate-binding protein & $\ldots$ & 4.7 & 2.6 & 0 & 0 & 12 \\
\hline blr6576 & Hypothetical protein & $\ldots$ & 17.2 & 22.9 & 0 & 0 & 50 \\
\hline
\end{tabular}


specifically transcribed. Likewise, rapidly degraded mRNAs might escape detection, whereas their more stable translation products do not. Another reason is that extraction was done from different biological material (i.e., whole nodules were used for RNA isolation and bacteroids were used for protein isolation). During bacteroid isolation, unstable proteins might have been lost. Nevertheless, the relaxed data set ought not to be neglected. It is a source of further potential functions involved in the adaptation of B. japonicum to the three hosts investigated. All of the 720 determinants were functionally classified according to the 14 categories established by the Kazusa DNA Research Institute (Kazusa website). Several categories were identified that are overrepresented with statistical significance (Supplementary Table S4). Particularly eyecatching are the high numbers of hypothetical proteins (or genes) expressed in all three hosts.

\section{Concluding remarks.}

Recent developments in genome-wide expression analyses allow a systematic search for rhizobial determinants involved in symbiosis in an unbiased way. Here, we show the utility of an integrated transcriptomics-proteomics approach which has led to candidates for host-specific adaptation functions in $B$. japonicum, if one applies restrictive criteria in data analysis. Recently, the transcription profiles of $R$. leguminosarum bv. viciae in root nodules from pea and vetch were assessed; there was no change in response to these hosts except for a difference in 23S rRNA processing (Karunakaran et al. 2009). The identified host-specific adaptation genes and proteins are promising targets with which to study the concept of bacterial adaptation in greater detail and, thereby, unravel new aspects of bacteroid physiology. As a proof of principle, the blr1600 1604 operon, which had been detected as being host-specifically expressed in siratro, was found to be more relevant for the $B$. japonicum-siratro symbiosis than for the symbioses with soybean and cowpea. As the mutant phenotypes in this example showed, the adaptively responding genes may not be essential but at least important in the relevant host. The situation is somewhat reminiscent of the early events in the recognition and infection of legumes by rhizobia, in which few of the nod genes are essential, whereas most of the host-specificity nod genes are important but not essential for the nodulation process (Denarié et al. 1992; Spaink 2000).

\section{MATERIALS AND METHODS}

\section{DNA methods and construction of mutant strains.}

DNA was isolated from B. japonicum wild-type strain $110 s p c 4$ as previously described (Hahn and Hennecke 1984). Plasmid DNA from Escherichia coli strains was obtained by using the NucleoSpin Plasmid kit (Macherey-Nagel, Düren, Germany). Recombinant DNA work was performed according to standard protocols (Sambrook and Russel 2001). To construct a deletion mutation in the blr1600-1604 gene cluster, polymerase chain reaction (PCR) fragments of the $5^{\prime}$ and $3^{\prime}$ flanking regions of the blr1600-1604 genes were amplified using the following primer pairs: blr1600-3_rev (GGACTAGT CCCGAATCAACACGTAGG) and blr1600-4_for (TTTCTGC AGCACATGCGGTTCGTGTAGAGG) for the $5^{\prime}$ region and blr1600-5_rev (TTTCTGCAGCTTCCAGGCGGCATAAAAT TTC) and blr1600-6_for (CCGCTCGAGTAGCGATCGCCGA GAGGCGAG) for the $3^{\prime}$ region. PCR products were cloned in the pGEM-T Easy vector (Promega Corp., Madison, WI, U.S.A.) and, subsequently, in pSUP202pol2 vector lacking the cat resistance cassette. A 1.2-kb kanamycin resistance cassette (aphII) derived from pBSL15 (Alexeyev 1995) was introduced in both directions between the up- and downstream regions.
The resulting plasmids pRJ6202 and pRJ6203 were transferred into B. japonicum strain 110 spc 4 for marker replacement using previously described methods (Hahn and Hennecke 1984). The correct genomic structure was verified by PCR. The resulting deletion mutants were named 6202 and 6203 (Fig. 3A). They still had coding information for $23 \mathrm{~N}$-terminal amino acids (blr1600) and 15 C-terminal amino acids (blr1604).

\section{Plant material, inoculation, and growth conditions.}

Soybean seed (G. $\max ($ L.) Merr. cv. Williams) was supplied by CIFA, Las Torres-Tomejil (Seville, Spain) and surface sterilized as described (Hahn and Hennecke 1984; Göttfert et al. 1990). Cowpea (V. unguiculata (L.) Walp. cv. Red Caloona) and siratro (Macroptilium atropurpureum (DC.) Urb.) seed were provided by W. D. Broughton (University of Geneva, Switzerland) and surface sterilized according to Lewin and associates (1990). Germination, inoculation, and growth of the plants were done as previously described (Hahn and Hennecke 1984; Göttfert et al. 1990). Nitrogenase activity in root nodules was determined at $13,21,31$, and 43 days postinoculation (dpi) and found to be maximal at $21 \mathrm{dpi}$ for cowpea (similar to what was observed earlier for soybean) (Pessi et al. 2007) and 31 dpi for siratro, which might explain the slower development of siratro plants compared with the other hosts. These time points were chosen for transcriptome and proteome analyses in at least three independent experiments (described below). The acetylene-dependent ethylene production was measured to monitor nitrogenase activity (Hahn and Hennecke 1984; Göttfert et al. 1990).

\section{Transcriptome analyses.}

Root nodules collected from plants at the time of maximal nitrogenase activity were immediately frozen in liquid nitrogen and stored at $-80^{\circ} \mathrm{C}$ for subsequent RNA isolation. RNA from symbiotically grown bacteria was extracted and processed as previously described (Pessi et al. 2007). Three to four biological replicates of $B$. japonicum-infected root nodules from cowpea and siratro were prepared for transcriptome analyses. The data set for soybean was taken from Pessi and associates (2007). Independently, host root-only tissue was collected and processed for GeneChip analysis and used as hybridization control (described below) (Pessi et al. 2007).

High-density oligonucleotide GeneChip (BJAPETHa520090), custom-designed and manufactured by Affymetrix (Santa Clara, CA, U.S.A.), was used for transcriptome profiling (Hauser et al. 2007). All probe pairs had been pruned against the soybean expressed sequence tags represented on the commercial Affymetrix Soybean Genome Array to minimize cross hybridization with plant-derived material in total RNA extracted from nodules. Furthermore, the chip includes 29 host control genes for soybean (including the 18S rRNA), and 4 for cowpea (Hauser et al. 2007). Hybridization signals were preprocessed by employing the Gene Chip Operating System software from Affymetrix. Signals were scaled to a target signal intensity of 500 for all arrays. Data analysis was performed using GeneSpring GX 7.3.1 software (Agilent Technologies, Palo Alto CA, U.S.A.). Each chip belonging to one experiment was normalized to the median. Values below 0.01 were set to 0.01 . As described previously, stringent criteria were applied to minimize false positive results among the bacteroid-induced genes (Pessi et al. 2007). This included filtering on flags (present in at least two out of three of the arrays) and root filtering, in which the signal in bacteroids was requested to be at least two times higher than in the control hybridizations with rootonly material. The amount of plant RNA was normalized to the signal of the G. $\max 18 \mathrm{~S}$ rRNA. Genes differentially expressed in siratro, soybean, and cowpea were selected using an analy- 
sis of variance with a post-hoc Tukey test. A subsequent filter (twofold cutoff) for the fold change was applied. Differentially expressed genes were clustered hierarchically using GeneSpring 7.3.1 (Fig. 1). The Euclidean distance was used as distance measure between two gene expression profiles, and clusters were aggregated using the complete linkage algorithm. Functional classification of the differentially expressed genes was based on Kazusa functional gene classification for $B$. japonicum (Kazusa website) which comprises 14 major categories. To identify functional groups that are significantly overor underrepresented among the differentially expressed genes, Fisher's Exact Test was used with a $P$ value threshold of 0.01 .

\section{Proteome analyses.}

Notably, the extraction procedure differed between the transcriptomics and proteomics experiments. Total nodule RNA was extracted and used for GeneChip hybridizations, whereas bacteroids were extracted and fractionated prior to proteome analyses as described recently (Delmotte et al. 2010). Protein data for soybean nodules were taken from our previous study (Delmotte et al. 2010) whereas protein data for cowpea and siratro nodules were generated within this study. To this end, between 1 and $4.38 \mathrm{~g}$ of root nodules (which were collected at the peak of maximal nitrogenase activity) from at least 40 plants infected with the $B$. japonicum wild type were used per biological replicate. Bacteroid isolation from nodules and bacteroid fractionation were done essentially as described by Sarma and Emerich (2005), except that cells were collected by centrifugation at higher $g$ value $(8,000 \times g$ for $10 \mathrm{~min})$. Proteins were then extracted using the AllPrep DNA/RNA/Protein Mini Kit according to the instructions in the kit manual (Qiagen, Hilden, Germany). Proteins were separated on a Tris$\mathrm{HCl}$ polyacrylamide gel (10.5-to- $14 \%$ linear gradient, 13.3 by $8.7 \mathrm{~cm}$ ) obtained from Bio-Rad Laboratories (Reinach, Switzerland), and staining was performed with Coomassie blue. Gel lanes were cut in 20 pieces and destained. After reduction and carbamidomethylation, proteins were digested with trypsin (Promega Corp.). Digestion was quenched, and peptides were cleaned up with a C18 ZipTip supplied by Millipore Corporation (Billerica MA, U.S.A.).

Peptides were separated by reverse-phase high-performance liquid chromatography, and mass spectrometric detection was performed with LTQ-Orbitrap mass spectrometers (Thermo Fisher Scientific, Waltham MA, U.S.A.). The four most abundant double- or triple-charged ions from the high-accuracy survey scan with a minimum ion count of 500 were automatically taken for further MS/MS analysis at the linear ion trap. Precursor masses already taken for MS/MS were excluded for further selection for $60 \mathrm{~s}$. All mass spectra were recorded in positive ion mode with an electrospray source voltage between 1.5 and $1.90 \mathrm{kV}$. Precursor mass spectra were acquired at the Orbitrap mass analyzer with a scan range from $\mathrm{m} / z 300.0$ to $1,600.0$ using internal lock mass calibration on $\mathrm{m} / \mathrm{z}, 429.088735$ and 445.120025. Resolution was set to 60,000 at $\mathrm{m} / \mathrm{z} \quad 400$ (Delmotte et al. 2009).

Mass spectra processing was performed with Xcalibur 2.0.7 (Thermo Fisher Scientific). Peak list generation for database searches was performed with Mascot Distiller 2.1.1.0 (Matrix Science, London). A protein database was built by combining protein sequences of $B$. japonicum USDA 110 (8,317 protein sequences downloaded from the National Center for Biotechnology Information [NCBI] website) with protein sequences of G. $\max (62,199$ sequences downloaded from the soybean annotation website). In all, 258 protein sequences of usual contaminants (e.g., human keratin and trypsin) were also appended to the database. All experimental mass lists were tested against a composite version of the database, created by concatenating the target protein sequences with reversed sequences (total of 141,290 sequences, target-decoy searches) as described by Elias and Gygi (2007). Database searches were performed with Mascot 2.2 (Perkins et al. 1999) (Matrix Science) and X!Tandem (Craig and Beavis 2004). Results were validated with Scaffold 2.02.01 (Proteome Software Inc., Portland, OR, U.S.A.). Peptide identifications were accepted if they could be established at greater than $95 \%$ probability as specified by the Peptide Prophet algorithm (Keller et al. 2002). Probability greater than $99 \%$-in sense of the Protein Prophet algorithm (Nesvizhskii et al. 2003)—was required to validate protein identifications. One-hit wonders were removed (i.e., only proteins identified with at least two detected peptides were considered), and proteins that contained similar peptides and could not be differentiated based on MS/MS analysis alone were grouped to satisfy the principle of parsimony. The computed false discovery rate at the spectrum level was below $0.02 \%$. The complete list of 2,633 B. japonicum proteins that were identified during symbiosis with cowpea, siratro, or soybean is provided as Supplementary Table S5. If only those proteins detected in two out of three biological samples are considered as positive hits, then 2,015 proteins were identified.

Two programs from the Expasy website, TMHMMv2.0 and SignalPv3.0, were used to predict transmembrane-spanning domains and signal peptides of secreted proteins, respectively.

\section{ACKNOWLEDGMENTS}

We thank R. Schlapbach for access to the Functional Genomics Center Zurich to perform the transcriptomics and proteomics experiments; D.-N. Rodríguez-Navarro and F. Temprano (Las Torres-Tomejil, Seville, Spain) for providing soybean seed; and W. Broughton (University of Geneva, Switzerland) for cowpea and siratro seeds.

\section{LITERATURE CITED}

Alexeyev, M. F. 1995. Three kanamycin resistance gene cassettes with different polylinkers. Biotechniques 18:52-56.

Brito, B., Toffanin, A., Prieto, R.-I., Imperial, J., Ruiz-Argueso, T., and Palacios, J. M. 2008. Host-dependent expression of Rhizobium leguminosarum bv. viciae hydrogenase is controlled at transcriptional and post-transcriptional levels in legume nodules. Mol. Plant-Microbe Interact. 21:597-604.

Broughton, W. J., Jabbouri, S., and Perret, X. 2000. Keys to symbiotic harmony. J. Bacteriol. 182:5641-5652.

Craig, R., and Beavis, R. C. 2004. TANDEM: Matching proteins with tandem mass spectra. Bioinformatics 20:1466-1467.

Delgado-Salinas, A., Bibler, R., and Lavin, M. 2006. Phylogeny of the genus Phaseolus (Leguminosae): A recent diversification in an ancient landscape. Syst. Bot. 31:779-791.

Delmotte, N., Knief, C., Chaffron, S., Innerebner, G., Roschitzki, B. Schlapbach, R., von Mering, C., and Vorholt, J. A. 2009. Community proteogenomics reveals insights into the physiology of phyllosphere bacteria. Proc. Natl. Acad. Sci. U.S.A. 106:16428-16433.

Delmotte, N., Ahrens, C. H., Knief, C., Qeli E., Koch, M., Fischer, H. M., Volholt, J. A., Hennecke H., and Pessi, G. 2010. An integrated proteomics and transcriptomics reference dataset provides new insights into the Bradyrhizobium japonicum bacteroid metabolism in soybean root nodules. Proteomics. DOI: 10.1002/pmic.200900710. Published online.

Denarié, J., Debelle, F., and Rosenberg, C. 1992. Signaling and host range variation in nodulation. Annu. Rev. Microbiol. 46:497-531.

Denarié, J., Debelle, F., and Promé, J. C. 1996. Rhizobium lipo-chitooligosaccharide nodulation factors: Signaling molecules mediating recognition and morphogenesis. Annu. Rev. Biochem. 65:503-535.

Ehrhardt, D. W., Atkinson, E. M., Faull, K. F., Freedberg, D. I., Sutherlin, D. P., Armstrong, R., and Long, S. R. 1995. In vitro sulfotransferase activity of NodH, a nodulation protein of Rhizobium meliloti required for host-specific nodulation. J. Bacteriol. 177:6237-6245.

Elias, J. E., and Gygi, S. P. 2007. Target-decoy search strategy for increased confidence in large-scale protein identifications by mass spectrometry. Nat. Methods 4:207-214.

Göttfert, M., Grob, P., and Hennecke, H. 1990. Proposed regulatory pathway encoded by the nodV and nodW genes, determinants of host speci- 
ficity in Bradyrhizobium japonicum. Proc. Natl. Acad. Sci. U.S.A. 87:2680-2684.

Hahn, M., and Hennecke, H. 1984. Localized mutagenesis in Rhizobium japonicum. Mol. Gen. Genet. 193:46-52.

Hauser, F., Pessi, G., Friberg, M., Weber, C., Rusca, N., Lindemann, A., Fischer, H. M., and Hennecke, H. 2007. Dissection of the Bradyrhizobium japonicum $\mathrm{Nif} A+\sigma^{54}$ regulon, and identification of a ferredoxin gene $(f d x N)$ for symbiotic nitrogen fixation. Mol. Genet. Genomics 278:255-271

Higgins, C. F. 2001. ABC transporters: Physiology, structure and mechanism - an overview. Res. Microbiol. 152:205-210.

Kaneko, T., Nakamura, Y., Sato, S., Minamisawa, K., Uchiumi, T., Sasamoto, S., Watanabe, A., Idesawa, K., Iriguchi, M., Kawashima, K., Kohara, M., Matsumoto, M., Shimpo, S., Tsuruoka, H., Wada, T., Yamada, M., and Tabata, S. 2002. Complete genomic sequence of nitrogen-fixing symbiotic bacterium Bradyrhizobium japonicum USDA110. DNA Res. 9:189-197.

Karunakaran, R., Ramachandran, V. K., Seaman, J. C., East, A. K., Mouhsine, B., Mauchline, T. H., Prell, J., Skeffington, A., and Poole, P. S. 2009. Transcriptomic analysis of Rhizobium leguminosarum biovar viciae in symbiosis with host plants Pisum sativum and Vicia cracca. J. Bacteriol. 191:4002-4014.

Keller, A., Nesvizhskii, A. I., Kolker, E., and Aebersold, R. 2002. Empirical statistical model to estimate the accuracy of peptide identifications made by MS/MS and database search. Anal. Chem. 74:5383-5392.

Krusell, L., Krause, K., Ott, T., Desbrosses, G., Kramer, U., Sato, S., Nakamura, Y., Tabata, S., James, E. K., Sandal, N., Stougaard, J., Kawaguchi, M., Miyamoto, A., Suganuma, N., and Udvardi, M. K. 2005. The sulfate transporter SST1 is crucial for symbiotic nitrogen fixation in Lotus japonicus root nodules. Plant Cell 17:1625-1636.

Lee, J., and Hymowitz, T. 2001. A molecular phylogenetic study of the subtribe Glycininae (Leguminosae) derived from the chloroplast DNA rps 16 intron sequences. Am. J. Bot. 88:2064-2073

Lewin, A., Cervantes, E., Chee-Hoong, W., and Broughton, W. J. 1990. nodSU, two new nod genes of the broad host range Rhizobium strain NGR234 encode host-specific nodulation of the tropical tree Leucaena leucocephala. Mol. Plant-Microbe Interact. 3:317-326.

Loh, J., Garcia, M., and Stacey, G. 1997. NodV and NodW, a second flavonoid recognition system regulating nod gene expression in Bradyrhizobium japonicum. J. Bacteriol. 179:3013-3020.

Madsen, E. B., Madsen, L. H., Radutoiu, S., Olbryt, M., Rakwalska, M., Szczyglowski, K., Sato, S., Kaneko, T., Tabata, S., Sandal, N., and Stougaard, J. 2003. A receptor kinase gene of the LysM type is involved in legume perception of rhizobial signals. Nature 425:637-640.

McIver, J., Djordjevic, M. A., Weinman, J. J., Bender, G. L., and Rolfe, B. G. 1989. Extension of host range of Rhizobium leguminosarum bv. trifolii caused by point mutations in nodD that result in alterations in regulatory function and recognition of inducer molecules. Mol. PlantMicrobe Interact. 2:97-106.

Milcamps, A., and de Bruijn, F. J. 1999. Identification of a novel nutrientdeprivation-induced Sinorhizobium meliloti gene $(h m g A)$ involved in the degradation of tyrosine. Microbiology 145:935-947.

Miller, S. H., Elliot, R. M., Sullivan, J. T., and Ronson, C. W. 2007. Hostspecific regulation of symbiotic nitrogen fixation in Rhizobium leguminosarum biovar trifolii Microbiology 153:3184-3195.

Moussatova, A., Kandt, C., O'Mara, M. L., and Tieleman, D. P. 2008.
ATP-binding cassette transporters in Escherichia coli. Biochim. Biophys. Acta 1778:1757-1771.

Moutran, A., Balan, A., Ferreira, L. C., Giorgetti, A., Tramontano, A., and Ferreira, R. C. 2007. Structural model and ligand interactions of the Xanthomonas axonopodis pv. citri oligopeptide-binding protein. Genet. Mol. Res. 6:1169-1177.

Nesvizhskii, A. I., Keller, A., Kolker, E., and Aebersold, R. 2003. A statistical model for identifying proteins by tandem mass spectrometry. Anal. Chem. 75:4646-4658.

Oke, V., and Long, S. R. 1999. Bacteroid formation in the Rhizobium-legume symbiosis. Curr. Opin. Microbiol. 2:641-646.

Osteras, M., Finan, T. M., and Stanley, J. 1991. Site-directed mutagenesis and DNA sequence of pckA of Rhizobium NGR234, encoding phosphoenolpyruvate carboxykinase: Gluconeogenesis and host-dependent symbiotic phenotype. Mol. Gen. Genet. 230:257-269.

Perkins, D. N., Pappin, D. J., Creasy, D. M., and Cottrell, J. S. 1999. Probability-based protein identification by searching sequence databases using mass spectrometry data. Electrophoresis 20:3551-3567.

Perret, X., Staehelin, C., and Broughton, W. J. 2000. Molecular basis of symbiotic promiscuity. Microbiol. Mol. Biol. Rev. 64:180-201.

Pessi, G., Ahrens, C. H., Rehrauer, H., Lindemann, A., Hauser, F., Fischer, H.-M., and Hennecke, H. 2007. Genome-wide transcript analysis of Bradyrhizobium japonicum bacteroids in soybean root nodules. Mol. Plant-Microbe Interact. 20:1353-1363.

Peters, N. K., Frost, J. W., and Long, S. R. 1986. A plant flavone, luteolin, induces expression of Rhizobium meliloti nodulation genes. Science 233:977-980.

Prell, J., and Poole, P. 2006. Metabolic changes of rhizobia in legume nodules. Trends Microbiol. 14:161-168.

Sambrook, J., and Russel, D. W. 2001. Molecular Cloning: A Laboratory Manual. Cold Spring Harbor Laboratory Press, Cold Spring Harbor, NY, U.S.A.

Sarma, A. D., and Emerich, D. W. 2005. Global protein expression pattern of Bradyrhizobium japonicum bacteroids: A prelude to functional proteomics. Proteomics 5:4170-4184.

Schell, M. A. 1993. Molecular biology of the LysR family of transcriptional regulators. Annu. Rev. Microbiol. 47:597-626.

Spaink, H. P. 2000. Root nodulation and infection factors produced by rhizobial bacteria. Annu. Rev. Microbiol. 54:257-288.

Tam, R., and Saier, M. H., Jr. 1993. Structural, functional, and evolutionary relationships among extracellular solute-binding receptors of bacteria. Microbiol. Rev. 57:320-346.

Zhang, H., Herman, J. P., Bolton, H., Jr., Zhang, Z., Clark, S., and Xun, L. 2007. Evidence that bacterial ABC-type transporter imports free EDTA for metabolism. J. Bacteriol. 189:7991-7997.

\section{AUTHOR-RECOMMENDED INTERNET RESOURCES}

The ExPASy (Expert Protein Analysis System) proteomics server: www.expasy.ch

Kazusa DNA Research Institute website: www.kazusa.or.jp/e/index.html

NCBI home page: www.ncbi.nlm.nih.gov

NCBI Gene Expression Omnibus (GEO) database:

www.ncbi.nlm.nih.gov/geo

Soybean annotation database: ftp://ftp.jgi-psf.org/pub/JGI_data 